\title{
REVIEW
}

\section{Efficacy and tolerability of intralesional bleomycin in dermatology: A systematic review}

\author{
Liora Bik, MD, ${ }^{\mathrm{a}}$ Tobias Sangers, MD, ${ }^{\mathrm{a}}$ Karin Greveling, MD, PhD, ${ }^{\mathrm{a}}$ Errol Prens, MD, PhD, ${ }^{\mathrm{a}}$ \\ Merete Haedersdal, MD, PhD, DMSc, ${ }^{b}$ and Martijn van Doorn, $\mathrm{MD}, \mathrm{PhD}^{\mathrm{a}}$ \\ Rotterdam, the Netberlands; and Copenhagen, Denmark
}

\begin{abstract}
Bleomycin is widely used as an off-label treatment for various dermatologic indications. However, a much-needed critical appraisal of the currently available evidence is lacking. We therefore evaluated the quality of clinical evidence for the efficacy and safety of intralesional bleomycin treatment for dermatologic indications with the aim to provide evidence-based recommendations for clinical practice. The PubMed, Embase, Medline Ovid, Web of Science, Cochrane Central, and Google Scholar databases were systematically searched. Two authors independently selected relevant studies according to predefined inclusion and exclusion criteria. We assessed the methodologic quality with the Cochrane Collaboration risk-of-bias assessment tool and selected 10 randomized clinical trials and 15 clinical controlled trials. Treatment indications included common warts, nonmelanoma skin cancer, cutaneous metastases, keloid and hypertrophic scars, and hemangioma. Intralesional bleomycin treatment showed significantly higher cure rates for warts compared with other treatments. Local adverse events included erythema, blackening, eschar formation, and superficial ulceration. None of the studies reported systemic adverse events. Methodologic quality of the studies was generally low. Consequently, no firm recommendations can be made for intralesional bleomycin treatment in clinical practice. However, this review suggests that intralesional bleomycin is a successful and well-tolerated treatment for recalcitrant warts. ( $\mathrm{J}$ Am Acad Dermatol https://doi.org/10.1016/j.jaad.2020.02.018.)
\end{abstract}

Key words: bleomycin; cutaneous metastases; dermatology; drug response; efficacy; electrochemotherapy; hemangioma; hypertrophic scars; intralesional; keloid; nonmelanoma skin cancer; safety; systematic review.

B leomycin has been approved as cytostatic drug for the treatment of squamous cell carcinoma of head, neck, and external genitalia in dermatology. ${ }^{1,2}$ In clinical practice, however, bleomycin is also used off-label for various other dermatologic indications.

Bleomycin is an antineoplastic antibiotic derived from Streptomyces verticillus. ${ }^{3}$ Multiple subtypes (A1-6 and B1-5) are available, of which bleomycin $\mathrm{A} 2$ and $\mathrm{B} 2$ are most commonly used in clinical practice. The latter subtypes are hydrophilic and have a metal binding core that is the key factor in the mechanism of action. Bleomycin is primarily eliminated by renal excretion and to a lesser extent by the bleomycin hydrolase (BMH) enzyme. ${ }^{4} \mathrm{BMH}$

\footnotetext{
From the Department of Dermatology, Erasmus MC University Medical Center, Rotterdam ${ }^{\text {a }}$; and the Department of Dermatology, Bispebjerg Hospital, University of Copenhagen. ${ }^{\text {b }}$ Funding sources: None.

Conflicts of interest: None disclosed. IRB approval status: Not applicable. Accepted for publication February 6, 2020.

Reprints not available from the authors.
}

shows the highest activity in bone marrow and is least active in the lungs and skin, with consequently more bleomycin-related toxicity in the latter tissues. ${ }^{5}$

Bleomycin's main mechanism of action is DNA-strand scissoring. Specifically, in the presence of molecular oxygen, it can oxidize metal ions such as $\mathrm{Fe}^{2+}$ to $\mathrm{Fe}^{3+}$, creating free radicals. Bleomycin binds to DNA by an electrostatic attraction and breaks the DNA backbone, which ultimately ends in cell cycle arrest. ${ }^{5-7}$ Bleomycin administered to the skin results in apoptosis of keratinocytes, sclerosing of endothelium cells, and inhibition of collagen synthesis. ${ }^{8}$

Severe adverse reactions have been reported after intravenous chemotherapy with bleomycin. Pulmonary fibrosis has been the most serious

\footnotetext{
Correspondence to: Liora Bik, MD, Erasmus MC, Department of Dermatology, Doctor Molewaterplein 40, 3015 GD Rotterdam, the Netherlands. E-mail: I.bik@erasmusmc.nl.

Published online March 18, 2020.

0190-9622/\$36.00

(C) 2020 by the American Academy of Dermatology, Inc.

https://doi.org/10.1016/j.jaad.2020.02.018
} 
reported adverse reaction after a total dose exceeding $>400$ U. ${ }^{9,10}$ Severe cutaneous toxicity has been reported after cumulative dosages of 200 to $300 \mathrm{U}$, including scleroderma, neutrophilic eccrine hidradenitis, and acute generalized exanthematous pustulosis. ${ }^{10-13}$

In dermatology, bleomycin is mainly used as an intralesional treatment, and the dosage usually does not exceed 2 to $6 \mathrm{U}$ per session. ${ }^{14}$ Sporadic reported adverse reactions after intralesional administration include gangrene, onychodystrophy, Raynaud phenomenon, scleroderma, and flagellate erythema. ${ }^{11,15-18}$ Local skin reactions after bleomycin injections include transient symptoms of erythema, edema, blackening, eschar formation, pain, and pigmentary changes. ${ }^{19,20}$

Contraindications for bleomycin include pregnancy, Raynaud phenomenon, peripheral vascular diseases, and bleomycin intolerance. ${ }^{21}$

The efficacy and safety of intralesional bleomycin treatment for dermatologic indications has been investigated in various clinical trials. To date, however, a much-needed critical appraisal of the currently available evidence has been lacking. The objective of this study was to systematically review and evaluate the quality of clinical evidence for the efficacy and safety of intralesional bleomycin treatment for dermatologic indications and to provide evidencebased recommendations for clinical practice.

\section{METHODS}

In January 2019, we conducted a systematic literature search for intralesional bleomycin treatment for dermatologic indications. Relevant keywords were used to search in PubMed, Embase, Medline Ovid, Web of Science, Cochrane Central, and Google Scholar. The systematic review was registered in PROSPERO (CRD42019131934) and followed the Preferred Reporting Items for Systematic Reviews and Meta-Analyses (PRISMA) guidelines for reporting systematic reviews.

The inclusion criteria for selection of the articles were English language, published from inception to January 2019, randomized controlled trials (RCTs), nonrandomized clinical controlled trials (CCTs), intralesional bleomycin treatments for dermatologic indications, and reporting clinical outcomes from intervention. Exclusion criteria were preclinical studies, monotherapy with intravenous or topical bleomycin, and case series with fewer than 10 patients. The primary outcome measure was efficacy, and the secondary outcome measure was safety.

Selection of the articles was performed independently by 2 authors (L.B. and T.S.). Articles were preselected based on the title and abstract. The final selection was based on full-text assessment. Reference lists of selected articles were screened for additional relevant studies. Standardized data extraction of the included studies was performed independently by 2 authors (L.B. and T.S.). The dosage of bleomycin was converted to United States Pharmacopeia units (U): $1 \mathrm{U}$ represents $1 \mathrm{mg}$ (by potency) or 1000 international units (IU). ${ }^{22}$

Methodologic quality was independently evaluated according to the Cochrane Collaborations risk-of-bias assessment tool. Disagreement between the 2 authors was resolved by discussion and involved a third author when necessary.

\section{RESULTS \\ Study characteristics}

The data search returned 2672 references. Duplicates were removed. The remaining 1647 references were screened based on title and abstract, whereupon 1531 references were excluded. Full texts of 116 references were obtained, and the inclusion and exclusion criteria were used to select 25 studies for this review (Fig 1).

The included studies are 10 RCTs and 15 CCTs published between 1979 and 2018, comprising a total of 1130 patients. The studies investigated intralesional bleomycin treatments for the following dermatologic indications: common warts, nonmelanoma skin cancer, cutaneous metastases, keloid, hypertrophic scars, hemangioma, and other indications. Described routes of administration included needle injections, jet injector, microneedling pen, multipuncture technique, and a bleomycin-covered microneedle patch.

\section{Common warts}

Fourteen studies with a total of 584 patients investigated intralesional bleomycin in 2657 
Abbreviations used:

RCT: randomized controlled trial

CCT: clinical controlled trial

PROSPERO: International prospective register of systematic reviews

PRISMA: transparent reporting of systematic reviews and meta-analyses

5-FU: 5-fluorouracil

TCA: Triamcinolone acetonide

common warts. Study characteristics and results are summarized in Table I. ${ }^{19,23-35}$

Intralesional bleomycin injections resulted in significantly higher complete cure rates than saline injection or cryotherapy $(P<.05){ }^{19,23-30}$ Administration of intralesional bleomycin via a microneedling pen showed comparable cure rates as intralesional bleomycin injections $(P=.474)$, but fewer patients reported pain $(20 \%$ vs $100 \%$; $P=.001){ }^{31}$ A bleomycin-covered microneedle patch resulted in comparable complete cure rates compared with cryotherapy but with significantly lower pain scores on a visual analog scale $(0.48 \pm 0.5$ vs $7.29 \pm 0.13 ; P<.0001){ }^{32}$

Use of a local electroporation procedure after bleomycin injections resulted in significantly higher complete cure rates than bleomycin alone $(P=.0015) .{ }^{33}$ During electroporation, electric pulses were generated with electrodes at the lesion site to increase cellular drug uptake.

One study showed significantly higher response rates in the placebo groups than in the group receiving bleomycin injections administered with a jet injector $(P=.018) .{ }^{34}$ The authors did not provide a specific explanation.

Patients who had received a transplant and were taking immunosuppressant drugs and patients without a transplant showed significantly higher complete cure rates for intralesional bleomycin injections than for placebo $(P<.0001)$. However, transplant recipients showed lower complete cure rates than patients without a transplant. ${ }^{27}$ No difference in complete cure rates was observed between different dosages of bleomycin injections (0.25, 0.50, or $1 \mathrm{U} / \mathrm{mL}$ ) (no $P$ value reported). ${ }^{35}$ Overall, plantar warts were more resistant to bleomycin treatment than warts on other anatomic locations. $^{19,25,26,35}$

\section{Nonmelanoma skin cancer}

One study including 113 patients investigated intralesional bleomycin with local electroporation in 113 nonmelanoma skin cancer tumors (Table II). ${ }^{14,20,36-44}$ This study showed a higher sustained complete response rate for electroporation with biphasic pulses of $50+50 \mu \mathrm{s}$ than for electroporation with biphasic pulses of $25+25 \mu$ s after intralesional bleomycin administration (no statistical test reported). ${ }^{36}$

\section{Cutaneous metastases}

Three studies including a total of 93 patients investigated intralesional bleomycin with local electroporation in 390 cutaneous metastases (Table II). Intralesional bleomycin, followed by local electroporation, showed significantly higher response rates in metastases of melanoma $(P=.017)$ compared with intralesional bleomycin alone $(P=.002){ }^{37,38}$ For cutaneous metastases of melanoma and nonmelanoma cancers, similar response rates have been reported for intralesional bleomycin, intravenous bleomycin, and intralesional cisplatin, all followed by local electroporation at the tumor site $(P=.09){ }^{39}$

\section{Keloid and hypertrophic scars}

Three studies with a total of 191 patients investigated intralesional bleomycin treatment in 191 keloids and hypertrophic scars (Table II). A significantly greater improvement on the Vancouver Scar Scale was reported with intralesional bleomycin compared with intralesional 5-fluorouracil (5-FU) or 5-FU combined with triamcinolone acetonide (TCA; $P<.005) .{ }^{14}$ Intralesional bleomycin showed improvement on the Patient and Observer Scar Assessment Scale comparable to TCA injections in patients with dark skin color (Fitzpatrick skin types III to $\mathrm{V}$; no $P$ value reported). ${ }^{20}$ Bleomycin multipuncture technique showed better resolution scores compared with cryotherapy combined with TCA injections $(P=.001){ }^{40}$ No recurrences were described after intralesional bleomycin treatment. $^{14,40}$

\section{Hemangioma}

Two studies with a total of 87 patients investigated intralesional bleomycin in 87 hemangiomas (Table II). Intralesional bleomycin showed significantly better response rates than intralesional TCA injections, but only in nonresponders to oral propranolol $(P=.037){ }^{41}$ No difference in hemangioma volume reduction was seen between intralesional bleomycin injections and oral propranolol in children (no statistical test reported). ${ }^{42}$

\section{Other indications}

Two studies with a total of 62 patients investigated 76 lesions of other indications for intralesional bleomycin treatment in dermatology (Table II). In 


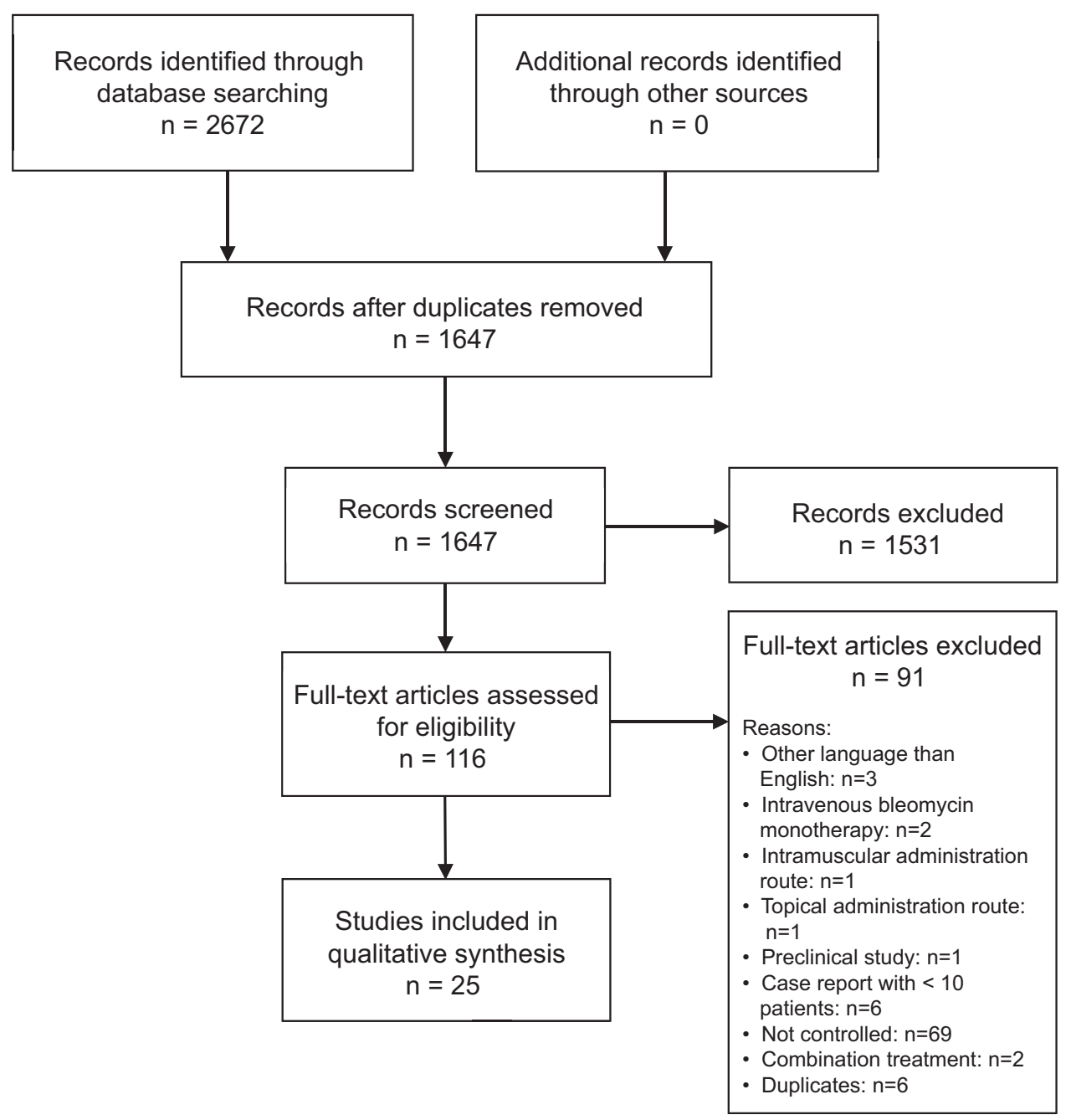

Fig 1. Flowchart of the exclusion process ending with 25 included studies.

corns, intralesional bleomycin injections after callus paring resulted in higher complete cure rates and pain reduction than callus paring alone (no $P$ value reported). ${ }^{43}$ In vulvar intraepithelial neoplasia, intralesional bleomycin injections and topical bleomycin application showed poor remission rates and high rates of disease progression (no statistical tests reported). ${ }^{44}$

\section{Reports of adverse events}

One study reported 3 cases of significant adverse events due to intralesional bleomycin treatment, but no details were described. ${ }^{29}$ Local skin reactions, including erythema, blackening, and eschar formation were described as part of the therapeutic effect of bleomycin.* Superficial ulceration was frequently reported but generally healed with no or minimal scarring. ${ }^{14,41,42,44}$ Hyperpigmentation after treatment

*References 19, 24, 26-28, 35, 37, 38, 43. was mainly observed in patients with dark skin types. ${ }^{14,20,40}$ Pain during or after the procedure, or both, was reported in 22 of 25 included studies. ${ }^{14,19,20,23-39,43,44}$ None of the included studies reported systemic adverse events.

\section{Methodologic quality of the included studies}

Most of the included studies had an unclear risk of bias for most of the methodologic criteria of the Cochrane Collaborations risk-of-bias assessment tool (Fig 2). Only 3 of the 25 included studies used high-quality randomization sequences, ${ }^{23,30,41}$ and all studies lacked concealment of allocation. Poor methodologic quality was found for blinding, incomplete outcome data, selective reporting, and other bias.

\section{DISCUSSION}

This systematic review provides an overview of the clinical studies investigating the efficacy and safety of intralesional bleomycin for dermatologic 
Table I. Characteristics and summary of results of included studies on intralesional bleomycin in common warts

\begin{tabular}{|c|c|c|c|c|c|c|c|c|c|c|c|c|c|}
\hline $\begin{array}{l}\text { First } \\
\text { author, } \\
\text { year }\end{array}$ & $\begin{array}{c}\text { Type of } \\
\text { lesion }\end{array}$ & $\begin{array}{l}\text { Study } \\
\text { design }\end{array}$ & $\begin{array}{c}\begin{array}{c}\text { No. of } \\
\text { patients } \\
\text { (lesions) }\end{array} \\
\end{array}$ & Intervention & Anesthetics & Frequency & $\begin{array}{c}\begin{array}{c}\text { Concentration \& } \\
\text { dosage }\end{array} \\
\end{array}$ & $\begin{array}{l}\text { Comparative } \\
\text { intervention }\end{array}$ & Results per patient & Results per lesion & $\begin{array}{c}\text { Follow-up } \\
\text { time }\end{array}$ & Adverse events & $\begin{array}{c}\text { Conflicts of } \\
\text { interest// } \\
\text { funding }\end{array}$ \\
\hline $\begin{array}{r}\text { Amer, }^{19} \\
1988\end{array}$ & $\begin{array}{r}\text { Resistant } \\
\text { warts }\end{array}$ & CCT & $38(143)$ & $\begin{array}{l}\text { Intralesional } \\
\text { bleomycin } \\
\text { injection }\end{array}$ & Not reported & $\begin{array}{l}\text { 1-2×, } \\
\text { interval of } \\
2 \text { weeks }\end{array}$ & $\begin{array}{l}1 \mathrm{U} / \mathrm{mL}, \leq 2 \mathrm{~mL} \\
\text { per treatment }\end{array}$ & $\begin{array}{l}\text { Placebo: } \\
\text { intralesional } \\
\text { saline injection } \\
\text { (in same } \\
\text { patient) }\end{array}$ & Not reported & $\begin{array}{l}\text { CR } 67.8 \% \text { vs } 2.9 \% \\
\text { and PR } 17.5 \% \\
\text { vs } 5.7 \% \text { resp. } \\
\text { bleomycin or } \\
\text { saline injection. } \\
\text { CR after } \\
\text { bleomycin } \\
\text { per location: } \\
77 \% \text { for hands } \\
\text { and feet, } 71.4 \% \\
\text { for periungual } \\
\text { warts, } 47.6 \% \text { for } \\
\text { plantar warts } \\
\text { (no statistical } \\
\text { tests) }\end{array}$ & $\begin{array}{l}\text { Not } \\
\quad \text { reported }\end{array}$ & $\begin{array}{l}\text { No systemic } \\
\text { adverse } \\
\text { events. } \\
\text { Local: pain } \\
\text { during } \\
\text { injection, } \\
\text { erythema, } \\
\text { blackening } \\
\text { and eschar } \\
\text { formation }\end{array}$ & Not reported \\
\hline $\begin{array}{c}\text { Barkat }^{23} \\
2018\end{array}$ & $\begin{array}{l}\text { Plantar } \\
\quad \text { warts }\end{array}$ & RCT & $46(46)$ & $\begin{array}{l}\text { Intralesional } \\
\text { bleomycin } \\
\text { injection }\end{array}$ & $\begin{array}{l}\text { Bleomycin } \\
\quad \text { diluted in } \\
\text { lidocaine } 2 \%\end{array}$ & $\begin{array}{l}\text { 1-4X, } \\
\text { interval of } \\
2 \text { weeks }\end{array}$ & $\begin{array}{c}1 \mathrm{U} / \mathrm{mL}, \leq 2 \mathrm{~mL} \text { per } \\
\text { treatment and } \\
\leq 1 \mathrm{~mL} \text { per wart }\end{array}$ & $\begin{array}{l}\text { Placebo: } \\
\quad \text { intralesional } \\
\text { saline } \\
\text { injection }\end{array}$ & $\begin{array}{l}\text { CR clinical and } \\
\text { dermoscopic } \\
\text { view, } 69.3 \% \\
\text { vs } 0 \% \text {; clinical } \\
\text { clearance but } \\
\text { dermoscopic } \\
\text { remnants of wart, } \\
19.2 \% \text { vs } 0 \% ; \\
\text { partial clinical } \\
\text { improvement, } \\
7.7 \% \text { vs } 5 \% \text {; } \\
\text { no response, } \\
3.8 \% \text { vs } 95 \% \\
\text { resp. bleomycin } \\
\text { or saline. } \\
(P<.0001) \text {. } \\
\text { Recurrence } \\
\text { rate } 0 \%\end{array}$ & Not reported & 3 months & $\begin{array}{l}\text { No systemic } \\
\text { adverse } \\
\text { events. } \\
\text { Local: pain } \\
\text { on first or } \\
\text { second day } \\
\text { after } \\
\text { injection }\end{array}$ & None declared \\
\hline $\begin{array}{c}\text { Shumack, }_{1}^{24} \\
1979\end{array}$ & $\begin{array}{c}\text { Common } \\
\text { warts }\end{array}$ & CCT & $?(1052)$ & $\begin{array}{l}\text { Intralesional } \\
\text { bleomycin } \\
\text { injection }\end{array}$ & $\begin{array}{l}\text { Local } \\
\text { anesthetic } \\
\text { injection } \\
\text { (Xylocaine } \\
1 \%)^{*}\end{array}$ & $\begin{array}{l}\text { 1-3 } \times \text {, interval of } \\
4 \text { weeks }\end{array}$ & $\begin{array}{r}1 \mathrm{U} / \mathrm{mL}, \leq 2 \mathrm{U} \text { per } \\
\text { patient in total }\end{array}$ & $\begin{array}{l}\text { Placebo: } \\
\text { intralesional } \\
\text { saline } \\
\text { injection }\end{array}$ & Not reported & $\begin{array}{l}\text { OR } 99.23 \% \\
\text { vs } 0 \% \text { resp. } \\
\text { bleomycin or } \\
\text { saline injection } \\
\text { (no statistical } \\
\text { tests) }\end{array}$ & $\begin{array}{l}\text { Not } \\
\quad \text { reported }\end{array}$ & $\begin{array}{l}\text { No systemic } \\
\text { adverse } \\
\text { events. } \\
\text { Local: pain, } \\
\text { blackening } \\
\text { thrombosis, } \\
\text { desiccating. } \\
\text { and } \\
\text { pigmentary } \\
\text { changes }\end{array}$ & Not reported \\
\hline $\begin{array}{c}\text { Bunney, }{ }^{25} \\
1984\end{array}$ & $\begin{array}{r}\text { Resistant } \\
\text { warts }\end{array}$ & RCT & $24(118)$ & $\begin{array}{l}\text { Intralesional } \\
\text { bleomycin } \\
\text { injection }\end{array}$ & Not reported & $\begin{array}{c}1-3 \times \text {, interval of } \\
3-6 \text { weeks }\end{array}$ & $\begin{array}{c}1 \mathrm{U} / \mathrm{mL}, \leq 0.2 \mathrm{~mL} \\
\text { per treatment } \\
\text { and } \leq 4 \mathrm{U} \\
\text { bleomycin } \\
\text { in total }\end{array}$ & $\begin{array}{l}\text { Placebo: } \\
\quad \text { intralesional } \\
\text { saline } \\
\text { injection }\end{array}$ & $\begin{array}{l}87.5 \% \text { showed } \\
\text { favorable } \\
\text { reaction to } \\
\text { bleomycin } \\
\text { than to saline. } \\
\text { CR in } 75 \% \text { of }\end{array}$ & $\begin{array}{l}\text { CR } 76 \% \text { vs } \\
10 \% \text { resp. } \\
\text { bleomycin } \\
\text { or saline }\end{array}$ & $>6$ months & $\begin{array}{l}\text { No systemic } \\
\text { adverse } \\
\text { event. } \\
\text { Local: pain }\end{array}$ & Not reported \\
\hline
\end{tabular}


Table I. Cont'd

\begin{tabular}{|c|c|c|c|c|c|c|c|c|c|c|c|c|c|}
\hline $\begin{array}{l}\text { First } \\
\text { author, } \\
\text { year }\end{array}$ & $\begin{array}{l}\text { Type of } \\
\text { lesion }\end{array}$ & $\begin{array}{l}\text { Study } \\
\text { design }\end{array}$ & $\begin{array}{c}\text { No. of } \\
\text { patients } \\
\text { (lesions) }\end{array}$ & Intervention & Anesthetics & Frequency & $\begin{array}{c}\text { Concentration \& } \\
\text { dosage }\end{array}$ & $\begin{array}{l}\text { Comparative } \\
\text { intervention }\end{array}$ & Results per patient & Results per lesion & $\begin{array}{l}\text { Follow-up } \\
\text { time }\end{array}$ & Adverse events & $\begin{array}{l}\text { Conflicts of } \\
\text { interest/ } \\
\text { funding }\end{array}$ \\
\hline & & & & & & & & & $\begin{array}{l}\text { resistant hand } \\
\text { warts and } 66 \% \\
\text { in mosaic } \\
\text { plantar warts }\end{array}$ & & & & \\
\hline $\begin{array}{c}\text { Shumer, }{ }^{26} \\
1983\end{array}$ & $\begin{array}{r}\text { Resistant } \\
\text { warts }\end{array}$ & $\begin{array}{l}\text { Cross- } \\
\text { over } \\
\text { CCT }\end{array}$ & $40(151)$ & $\begin{array}{l}\text { Intralesional } \\
\text { bleomycin } \\
\text { injection }\end{array}$ & Not reported & $\begin{array}{l}1-2 \times \text {, interval of } \\
2 \text { weeks }\end{array}$ & $\begin{array}{l}1 \mathrm{U} / \mathrm{mL} \text {, } \\
\quad \leq 2 \mathrm{~mL} \text { per } \\
\text { treatment }\end{array}$ & $\begin{array}{l}\text { Placebo: } \\
\text { intralesional } \\
\text { saline } \\
\text { injection } \\
\text { (switch to } \\
\text { other } \\
\text { intervention } \\
\text { after } 2 \text { failure } \\
\text { injections) }\end{array}$ & Not reported & $\begin{array}{l}\text { CR } 81 \% \text { vs } 0 \% \\
\text { resp. bleomycin } \\
\text { or saline } \\
\text { injection. } \\
\text { Specified CR: } \\
\text { plantar warts, } \\
60 \% ; \text {; periungual } \\
\text { warts, } 94 \% ; \\
\text { warts elsewhere } \\
\text { on extremities, } \\
95 \% . \text { Recurrence } \\
\text { rate } 0 \%\end{array}$ & 6-12 months & $\begin{array}{l}\text { No systemic } \\
\text { adverse } \\
\text { events. } \\
\text { Local: } \\
\text { hemorrhagic } \\
\text { eschar, pain, } \\
\text { erythema, } \\
\text { and swelling } \\
\text { for } 24-72 \\
\text { hours }\end{array}$ & Not reported \\
\hline $\begin{array}{r}\text { Sobh, }{ }^{27} \\
1991\end{array}$ & $\begin{array}{r}\text { Resistant } \\
\text { warts }\end{array}$ & CCT & $36(193)$ & $\begin{array}{l}\text { Intralesional } \\
\text { bleomycin } \\
\text { injection }\end{array}$ & Not reported & $\begin{array}{r}1-3 \times \text {, interval } \\
\text { of } 3 \text { weeks }\end{array}$ & $\begin{array}{l}1 \mathrm{U} / \mathrm{mL} \text {, } \\
\quad \leq 2 \mathrm{~mL} \text { per } \\
\text { treatment }\end{array}$ & $\begin{array}{l}\text { Placebo: } \\
\text { intralesional } \\
\text { saline } \\
\text { injection }\end{array}$ & Not reported & $\begin{array}{l}\text { Renal transplant } \\
\text { patients: CR } \\
37 \% \text { vs } 0 \% \text {, } \\
\text { PR } 3 \% \text { vs } 4 \% \\
\text { resp. bleomycin } \\
\text { or saline. } \\
\text { (P<.0001). } \\
\text { Nontransplant } \\
\text { patients: CR } \\
60 \% \text { vs } 2.5 \% \text {, } \\
\text { PR } 24 \% \text { vs } \\
2.5 \% \text { resp. } \\
\text { bleomycin } \\
\text { or saline. } \\
\text { (P }<.001) . \\
\text { Difference } \\
\text { between } \\
\text { patient } \\
\text { groups not } \\
\text { statistically } \\
\text { tested }\end{array}$ & $\begin{array}{l}\text { Not } \\
\text { reported }\end{array}$ & $\begin{array}{l}\text { No systemic } \\
\text { adverse } \\
\text { events. } \\
\text { Local: pain, } \\
\text { redness, } \\
\text { blackening } \\
\text { and eschar } \\
\text { formation }\end{array}$ & Not reported \\
\hline $\begin{array}{l}\text { Soni i, }^{28} \\
2011\end{array}$ & $\begin{array}{l}\text { Palmo- } \\
\text { plantar } \\
\text { and } \\
\text { periungual } \\
\text { warts }\end{array}$ & CCT & $50(157)$ & $\begin{array}{l}\text { Intralesional } \\
\text { bleomycin } \\
\text { injection }\end{array}$ & $\begin{array}{l}\text { Bleomycin } \\
\text { diluted with } \\
2 \% \text { lignocaine }\end{array}$ & $\begin{array}{r}1-2 \times \text {, interval } \\
\text { of } 2 \text { weeks }\end{array}$ & $\begin{array}{l}1 \mathrm{U} / \mathrm{mL}, \\
\quad \leq 2 \mathrm{~mL} \text { per } \\
\text { treatment }\end{array}$ & $\begin{array}{l}\text { Placebo: } \\
\text { intralesional } \\
\text { saline injection }\end{array}$ & Not reported & $\begin{array}{l}\text { CR } 96.47 \% \text { vs } \\
11.11 \% \text { resp. } \\
\text { bleomycin } \\
\text { or saline } \\
(P=.001) . \\
\text { Recurrence } \\
\text { rate } 0 \%\end{array}$ & 1 year & $\begin{array}{l}\text { No systemic } \\
\text { adverse } \\
\text { events. } \\
\text { Local: pain } \\
\text { and eschar } \\
\text { formation }\end{array}$ & None declared \\
\hline $\begin{array}{l}\text { Adalatkhah, } \\
2007\end{array}$ & $\begin{array}{l}\text { Common } \\
\text { warts } \\
\text { of hand } \\
\text { and feet }\end{array}$ & RCT & 52 (479) & $\begin{array}{l}\text { Intralesional } \\
\text { bleomycin } \\
\text { injection }\end{array}$ & $\begin{array}{l}\text { Bleomycin } \\
\text { diluted with } \\
2 \% \text { lidocaine }\end{array}$ & $\begin{array}{r}1-3 \times \text {, interval } \\
\text { of } 15 \text { days }\end{array}$ & $\begin{array}{l}0.5 \mathrm{U} / \mathrm{mL}, \\
\text { dosage not } \\
\text { reported }\end{array}$ & $\begin{array}{l}\text { Cryotherapy } \\
\text { (number of } \\
\text { freeze-thaw } \\
\text { cycles not } \\
\text { reported) }\end{array}$ & $\begin{array}{l}\text { CR } 86.4 \% \text { vs } \\
68.2 \% \text { resp. } \\
\text { bleomycin or } \\
\text { cryotherapy } \\
(P<.05) .\end{array}$ & $\begin{array}{l}\text { CR } 87.6 \% \text { vs } \\
72.3 \% \text { resp. } \\
\text { bleomycin or } \\
\text { cryotherapy } \\
(P<.001) . \text { RR }\end{array}$ & $\begin{array}{l}\text { Not } \\
\text { reported }\end{array}$ & $\begin{array}{l}\text { No systemic } \\
\text { adverse } \\
\text { events, } \\
\text { but } 3 \\
\text { unspecified }\end{array}$ & Not reported \\
\hline
\end{tabular}




\begin{tabular}{|c|c|c|c|c|c|c|c|c|c|c|c|c|c|}
\hline & & & & & & & & & $\begin{array}{l}\mathrm{RR}=1.27 \\
(1-1.6)\end{array}$ & $\begin{array}{l}\text { bleomycin }=1.23 \\
(1.22-1.33)\end{array}$ & & $\begin{array}{l}\text { cases of } \\
\text { significant } \\
\text { adverse } \\
\text { complications } \\
\text { due to } \\
\text { bleomycin } \\
\text { treatment. } \\
\text { Local: pain } \\
\text { No systemic }\end{array}$ & \\
\hline $\begin{array}{l}\text { Dhar, } \\
2009\end{array}$ & $\begin{array}{c}\text { Common } \\
\text { warts }\end{array}$ & RCT & $80(155)$ & $\begin{array}{l}\text { Intralesional } \\
\text { bleomycin } \\
\text { injection }\end{array}$ & $\begin{array}{l}\text { Bleomycin } \\
\quad \text { diluted with } \\
2 \% \text { lidocaine }\end{array}$ & $\begin{array}{r}\text { 1-4x, interval } \\
\text { of } 3 \text { weeks }\end{array}$ & 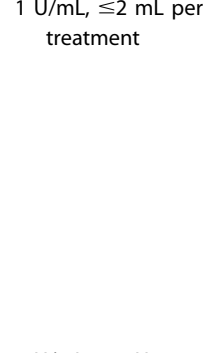 & $\begin{array}{l}\text { Cryotherapy, } \\
\text { double } \\
\text { freeze- } \\
\text { thaw } \\
\text { cycle }\end{array}$ & $\begin{array}{l}\text { CR } 94.9 \% \% \text { vs } \\
76.5 \% \text { resp. } \\
\text { bleomycin or } \\
\text { cryotherapy } \\
(P<.05 \text { and } \\
\text { RR }=7.67) . \\
\text { Treatment visits } \\
\text { needed } 1.38 \text { vs } \\
3.09 \text { resp. } \\
\text { bleomycin or } \\
\text { cryotherapy } \\
(P<.05) .\end{array}$ & $\begin{array}{l}\text { CR 97\% vs } \\
82 \% \text { resp. } \\
\text { bleomycin or } \\
\text { cryotherapy } \\
\text { (P<..55). } \\
\text { Recurrence } \\
\text { rate } 13 \% \text { v v } \\
23 \% \text { resp. } \\
\text { bleomycin or } \\
\text { cryotherapy } \\
\text { (not statistically } \\
\text { significant) }\end{array}$ & 8 weeks & $\begin{array}{l}\text { No systemic } \\
\text { adverse } \\
\text { events. } \\
\text { Local: pain } \\
\text { and } \\
\text { pigmentation } \\
\text { changes }\end{array}$ & None declared \\
\hline $\begin{array}{l}\text { Al-Nagar, }{ }^{31} \\
2018\end{array}$ & $\begin{array}{l}\text { Plantar } \\
\text { warts }\end{array}$ & CCT & $60(60)$ & $\begin{array}{l}\text { Intralesional } \\
\text { bleomycin } \\
\text { injection }\end{array}$ & $\begin{array}{l}\text { Bleomycin } \\
\text { diluted } \\
\text { with } 2 \% \\
\text { lidocaine }\end{array}$ & $\begin{array}{l}1-4 \times \text {, interval } \\
\text { of } 2 \text { weeks }\end{array}$ & $\begin{array}{c}1 \mathrm{U} / \mathrm{mL}, \leq 1 \text { U per } \\
\text { treatment }\end{array}$ & $\begin{array}{l}\text { Microneedling } \\
\text { pen with 2-mm } \\
\text { depth applied } \\
\text { for 2-3 minutes, } \\
\text { followed by } \\
\text { bleomycin } \\
\text { under } \\
\text { occlusion } \\
\text { for } 2 \text { hours } \\
\end{array}$ & $\begin{array}{l}\text { CR } 70 \% \text { vs } 83.3 \% \\
\text { and PR } 30 \% \\
\text { vs } 16.7 \% \text { resp. } \\
\text { bleomycin } \\
\text { injections or } \\
\text { microneedling } \\
\text { followed by } \\
\text { topical } \\
\text { bleomycin } \\
(P=.474) . \\
\text { Recurrence } \\
\text { rate } 0 \% \text { for both } \\
\text { groups. Pain } \\
\text { described } 100 \% \\
\text { vs } 20 \% \text { resp. } \\
\text { bleomycin } \\
\text { injection or } \\
\text { microneedling } \\
\text { and bleomycin } \\
(P=.001)\end{array}$ & Not reported & 6 months & $\begin{array}{l}\text { No systemic } \\
\text { adverse } \\
\text { events. } \\
\text { Local: pain, } \\
\text { erythema, } \\
\text { edema and } \\
\text { transient } \\
\text { induration }\end{array}$ & None declared \\
\hline $\begin{array}{l}\mathrm{Ryu}^{3,2} \\
2018\end{array}$ & $\begin{array}{c}\text { Common } \\
\text { warts }\end{array}$ & RCT & $42(42)$ & $\begin{array}{l}\text { Intralesional } \\
\text { bleomycin via } \\
\text { bleomycin- } \\
\text { coated } \\
\text { microneedle } \\
\text { patch }\end{array}$ & None & $\begin{array}{l}1 \times / d \text {, interval } \\
\text { of } 2 \text { weeks } \\
\text { until complete } \\
\text { clearance }\end{array}$ & $\begin{array}{l}518.12 \mu \mathrm{g} \text { of } \\
\text { bleomycin } \\
\text { on the surface } \\
\text { of microneedling } \\
\text { patch }\end{array}$ & $\begin{array}{l}\text { Cryotherapy, } \\
\text { double } \\
\text { freeze-thaw } \\
\text { cycle }\end{array}$ & $\begin{array}{l}\text { VAS score } \\
\text { mean } \pm \text { SD: } \\
0.48 \pm 0.5 \text { vs } \\
7.29 \pm 0.13 \text { resp. } \\
\text { bleomycyin or } \\
\text { cryotherapy } \\
(P<.0001)\end{array}$ & $\begin{array}{l}\text { CR } 61.90 \% \text { vs } \\
76.19 \% \text { resp. } \\
\text { bleomycin or } \\
\text { cryotherapy. } \\
\text { (statistical } \\
\text { tests not } \\
\text { reported). } \\
\text { Treatment } \\
\text { duration } \\
\text { until clearance } \\
14.0 \pm 6.6 \text { vs } \\
12.5 \pm 5.7 \text { resp. } \\
\text { bleomycin or }\end{array}$ & $\begin{array}{l}\text { Not } \\
\text { reported }\end{array}$ & $\begin{array}{l}\text { No systemic } \\
\text { adverse } \\
\text { events. } \\
\text { Local: pain, } \\
\text { erythema. } \\
\text { Scarring and } \\
\text { pigmentary } \\
\text { changes in } \\
\text { cryotherapy }\end{array}$ & None declared \\
\hline
\end{tabular}


Table I. Cont'd

\begin{tabular}{|c|c|c|c|c|c|c|c|c|c|c|c|c|c|}
\hline $\begin{array}{l}\text { First } \\
\text { author, } \\
\text { year }\end{array}$ & $\begin{array}{c}\text { Type of } \\
\text { lesion }\end{array}$ & $\begin{array}{l}\text { Study } \\
\text { design }\end{array}$ & $\begin{array}{c}\begin{array}{c}\text { No. of } \\
\text { patients } \\
\text { (lesions) }\end{array} \\
\text { (a) }\end{array}$ & Intervention & Anesthetics & Frequency & $\begin{array}{c}\text { Concentration \& } \\
\text { dosage }\end{array}$ & $\begin{array}{l}\text { Comparative } \\
\text { intervention }\end{array}$ & Results per patient & Results per lesion & $\begin{array}{c}\text { Follow-up } \\
\text { time }\end{array}$ & Adverse events & $\begin{array}{l}\text { Conflicts of } \\
\text { interest/ } \\
\text { funding }\end{array}$ \\
\hline & & & & & & & & & & $\begin{array}{l}\text { cryotherapy } \\
(P=.2771)\end{array}$ & & & \\
\hline $\begin{array}{c}\text { Pasqualit, }{ }^{33} \\
2017\end{array}$ & $\begin{array}{l}\text { Plantar } \\
\text { warts }\end{array}$ & CCT & $22(22)$ & $\begin{array}{l}\text { Intralesional } \\
\text { bleomycin } \\
\text { followed by } \\
\text { electroporation }\end{array}$ & $\begin{array}{l}\text { Local } \\
\quad \text { anesthetic } \\
\text { injection } \\
\text { (lidocaine) }\end{array}$ & 1 treatment & $\begin{array}{l}1 \mathrm{U} / \mathrm{cm}^{3} ; \\
\quad \leq 0.1 \mathrm{~mL} \text { per } \\
\text { injection }\end{array}$ & $\begin{array}{l}\text { Intralesional } \\
\quad \text { bleomycin } \\
\text { injection } \\
\text { alone }\end{array}$ & $\begin{array}{l}\text { CR } 78 \% \text { vs } 16 \% \\
\text { and PR } 22 \% \text { vs } \\
76 \% \text { resp. } \\
\text { bleomycin }+ \\
\text { electroporation } \\
\text { or bleomycin } \\
\text { alone after } \\
3 \text { months } \\
(P=.0015)\end{array}$ & $\begin{array}{l}\text { CR } 78 \% \text { vs } 16 \% \\
\text { and PR } 22 \% \text { vs } \\
76 \% \text { resp. } \\
\text { bleomycin }+ \\
\text { electroporation } \\
\text { or bleomycin } \\
\text { alone after } \\
3 \text { months } \\
(P=.0015)\end{array}$ & 3 months & $\begin{array}{l}\text { No systemic } \\
\text { adverse } \\
\text { event. } \\
\text { Local: pain, } \\
\text { redness, } \\
\text { tenderness, } \\
\text { skin } \\
\text { discoloration }\end{array}$ & $\begin{array}{l}\text { E.P. Spugnini } \\
\text { and A. } \\
\text { Baldi are } \\
\text { stockholders } \\
\text { in Biopulse } \\
\text { s.r.l. }\end{array}$ \\
\hline $\begin{array}{l}\text { Munkvad }^{34} \\
1983\end{array}$ & $\begin{array}{c}\text { Common } \\
\text { warts }\end{array}$ & RCT & $68(108)$ & $\begin{array}{l}\text { Intralesional } \\
\text { bleomycin } \\
\text { injection } \\
\text { administered } \\
\text { with a jet } \\
\text { injector }\end{array}$ & Not reported & $\begin{array}{r}1-4 \times \text {, interval } \\
\text { of } 2 \text { weeks }\end{array}$ & $\begin{array}{c}1 \mathrm{U} / \mathrm{mL}, \leq 0.9 \mathrm{U} \\
\text { in total }\end{array}$ & $\begin{array}{l}\text { Group II: } \\
\text { Intralesional } \\
\text { saline. } \\
\text { Group III: } \\
\text { intralesional } \\
\text { bleomycin } \\
\text { in oil. } \\
\text { Group IV: } \\
\text { intralesional } \\
\text { sesame oil } \\
\text { alone }\end{array}$ & Not reported & $\begin{array}{l}\text { CR } 18 \%, 42 \%, \\
23 \% \text { and } 46 \% \\
\text { resp. bleomycin } \\
\text { in saline, saline } \\
\text { alone, bleomycin } \\
\text { in oil or sesame- } \\
\text { oil alone. Placebo } \\
\text { treatments have } \\
\text { significant better } \\
\text { response rates } \\
\text { than active } \\
\text { treatments } \\
\text { with bleomycin } \\
(P=.018)\end{array}$ & 3 months & $\begin{array}{l}\text { No systemic } \\
\quad \text { adverse } \\
\text { events. } \\
\text { Local: } \\
\text { dullness, } \\
\text { pain, } \\
\text { swelling } \\
\text { or bleeding }\end{array}$ & Not reported \\
\hline $\begin{array}{r}\text { Hayes, }{ }^{35} \\
1986\end{array}$ & $\begin{array}{r}\text { Resistant } \\
\text { warts }\end{array}$ & RCT & $26(79)$ & $\begin{array}{l}\text { Intralesional } \\
\text { bleomycin } \\
\text { injection }\end{array}$ & Not reported & $\begin{array}{r}1-3 \times \text {, interval } \\
\text { of } 3 \text { weeks }\end{array}$ & $\begin{array}{l}0.25 \text { or } \\
\quad 0.5 \mathrm{U} / \mathrm{mL} \\
\leq 3 \mathrm{~mL} \text { per } \\
\text { treatment }\end{array}$ & $\begin{array}{l}\text { Bleomycin } \\
\quad \text { injection } \\
\text { of } 1 \mathrm{U} / \mathrm{mL}\end{array}$ & Not reported & $\begin{array}{l}\text { CR } 73.5 \%, \\
86.7 \%, 73.3 \% \\
\text { resp. 1, 0.5, and } \\
0.25 \mathrm{U} / \mathrm{mL} \\
\text { No statistical } \\
\text { difference. } \\
\text { Plantar warts } \\
\text { remained the } \\
\text { refractory. } \\
\text { Owing to small } \\
\text { number in } \\
0.25 \mathrm{U} / \mathrm{mL} \\
\text { group, no } \\
\text { assumptions } \\
\text { can be made } \\
\text { about the } \\
\text { effectivity. } \\
\text { Recurrence } \\
\text { rate } 0 \%\end{array}$ & 3 months & $\begin{array}{l}\text { No systemic } \\
\text { adverse } \\
\text { events. } \\
\text { Local: pain, } \\
\text { tenderness, } \\
\text { swelling, } \\
\text { erythema, } \\
\text { blackening, } \\
\text { eschar } \\
\text { formation, } \\
\text { and } \\
\text { horizontal } \\
\text { ridging } \\
\text { of nail } \\
\text { plate after } \\
\text { periungual } \\
\text { warts } \\
\text { injection }\end{array}$ & $\begin{array}{l}\text { Not } \\
\quad \text { reported }\end{array}$ \\
\hline
\end{tabular}

$C C T$, Clinical controlled trial; $C R$, complete response rate; No., number; $O R$, overall response rate (CR + PR); $P R$, partial response rate; $R C T$, randomized controlled trial; resp., respectively; $R R$, relative risk; U, United States Pharmacopeia unit; VAS, visual analog scale.

*Fresenius Kabi USA, Lake Zurich, Illinois. 
Table II. Characteristics and summary of results of included studies on intralesional bleomycin in nonmelanoma skin cancer, cutaneous metastases, keloids and hypertrophic scars, hemangioma, and other indications

\begin{tabular}{|c|c|c|c|c|c|c|c|c|c|c|c|c|c|}
\hline $\begin{array}{l}\text { First author, } \\
\text { year }\end{array}$ & $\begin{array}{c}\text { Type of } \\
\text { lesion }\end{array}$ & $\begin{array}{l}\text { Study } \\
\text { design }\end{array}$ & $\begin{array}{c}\text { No. of } \\
\text { patients } \\
\text { nations) } \\
\text { (lesions }\end{array}$ & Intervention & Anesthetics & Frequency & $\begin{array}{c}\text { Concentration } \\
\text { \& dosage }\end{array}$ & $\begin{array}{l}\text { Comparative } \\
\text { intervention }\end{array}$ & $\begin{array}{c}\text { Result per } \\
\text { patient }\end{array}$ & $\begin{array}{c}\text { Result per } \\
\text { lesion }\end{array}$ & $\begin{array}{l}\text { Follow- } \\
\text { up time }\end{array}$ & Adverse events & $\begin{array}{l}\text { Conflicts of } \\
\text { interest } / \\
\text { funding }\end{array}$ \\
\hline \multicolumn{14}{|l|}{$\begin{array}{l}\text { Nonmelanoma } \\
\text { skin cancer }\end{array}$} \\
\hline $\begin{array}{l}\text { Peycheva, }{ }^{36} \\
2004\end{array}$ & $\mathrm{BCC}$ and $\mathrm{SCC}$ & $\mathrm{CCT}$ & $113(113)$ & $\begin{array}{l}\text { Intralesional } \\
\text { bleomycin } \\
\text { injection } \\
\text { followed by } \\
\text { electroporation } \\
\text { sequence with } \\
\text { short pulses* }\end{array}$ & $\begin{array}{l}\text { Local anesthetic } \\
\text { injection } \\
\text { (lidocaine 1\%) }\end{array}$ & 1 treatment & $\begin{array}{l}\text { Concentration } \\
\text { not reported, } \\
0.5-0.8 \mathrm{U} \\
\text { depending } \\
\text { on tumor } \\
\text { size }\end{array}$ & $\begin{array}{l}\text { Intralesional } \\
\text { bleomycin } \\
\text { injections, } \\
\text { followed by } \\
\text { electroporation } \\
\text { sequence with } \\
\text { long pulses }{ }^{\dagger}\end{array}$ & $\begin{array}{l}\text { Initial CR of } 100 \% \\
\text { for both groups. } \\
\text { Sustained CR for } \\
\text { BCC } 80 \% \text { vs } 100 \% \text {, } \\
\text { and SCC } 71.4 \% \text { vs } \\
78.6 \% \text { resp. } \\
\text { electroporation } \\
\text { with short pulses } \\
\text { or long pulses } \\
\text { (no statistical } \\
\text { tests) }\end{array}$ & Not reported & 12 months & $\begin{array}{l}\text { No systemic of } \\
\text { serious adverse } \\
\text { events. Local: } \\
\text { reduced pain } \\
\text { described in } \\
\text { electroporation } \\
\text { group with } \\
\text { short pulses. }\end{array}$ & Not reported \\
\hline \multicolumn{14}{|l|}{$\begin{array}{l}\text { Cutaneous } \\
\text { metastases }\end{array}$} \\
\hline Gaudy, ${ }^{37} 2006$ & $\begin{array}{r}\text { Metastases of } \\
\text { melanoma }\end{array}$ & RCT & 12 (54) & $\begin{array}{l}\text { Intralesional } \\
\text { bleomycin } \\
\text { injection, } \\
\text { followed by } \\
\text { electroporation }\end{array}$ & $\begin{array}{l}\text { Local anesthetic } \\
\text { injection } \\
\text { (lidocaine 1\%+ } \\
\text { epinephrine) }\end{array}$ & 1 treatment & $\begin{array}{l}4 \mathrm{U} / \mathrm{mL}, \leq 40 \mathrm{U} \\
\text { of bleomycin } \\
\text { in total. }\end{array}$ & $\begin{array}{l}\text { Intralesional } \\
\text { bleomycin } \\
\text { alone }\end{array}$ & $\begin{array}{l}\text { Intention-to-treat } \\
\text { population } \\
\text { (n=12): } \\
\text { OR } 46 \% \text { vs } 25 \% \\
\text { resp. ECT or } \\
\text { bleomycin alone } \\
(P=.10) \text {. CR per } \\
\text { patient } 36 \% \\
\text { vs } 8 \% \text { resp. } \\
\text { bleomycin }+ \\
\text { electroporation } \\
\text { or bleomycin } \\
\text { alone }(P=.016)\end{array}$ & $\begin{array}{l}\text { CR per lesion } 64 \% \\
\text { vs } 18 \% \text { and } \\
\text { OR } 82 \% \text { vs } \\
54 \% \text { resp. } \\
\text { bleomycin }+ \\
\text { electroporation } \\
\text { or bleomycin } \\
\text { alone }(P=.017) \\
(P=.12)\end{array}$ & 24 weeks & $\begin{array}{l}\text { No systemic adverse } \\
\text { event. Local: pain, } \\
\text { muscle spasm } \\
\text { with myoclonia, } \\
\text { erythema, } \\
\text { edema and } \\
\text { necrosis }\end{array}$ & Not reported \\
\hline Byrne, $^{38} 2005$ & $\begin{array}{r}\text { Metastases of } \\
\text { melanoma }\end{array}$ & CCT & $19(46)$ & $\begin{array}{l}\text { Intralesional } \\
\text { bleomycin } \\
\text { injection, } \\
\text { followed by } \\
\text { electroporation }\end{array}$ & $\begin{array}{l}\text { Local anesthetic } \\
\text { injection } \\
\text { (1\% lignocaine }+ \\
\text { 1:100,000 } \\
\text { adrenaline), } \\
\text { mild oral } \\
\text { sedative or oral } \\
\text { analgesic }\end{array}$ & $\begin{array}{l}1-3 \times, \text { interval } \\
\text { not } \\
\text { reported }\end{array}$ & $\begin{array}{r}1 \mathrm{U} / \mathrm{mL}, \text { dosage } \\
\text { not reported }\end{array}$ & $\begin{array}{l}\text { Intralesional } \\
\quad \text { bleomycin } \\
\text { injection } \\
\text { alone }\end{array}$ & Not reported & $\begin{array}{l}\text { CR per lesion } \\
72 \% \text { vs } 26 \% \text {, } \\
\text { PR } 5 \% \text { vs } 5 \% \text {, } \\
\text { no response } \\
18 \% \text { vs } 15 \% \text {, } \\
\text { and disease } \\
\text { progression } \\
5 \% \text { vs } 53 \% \\
\text { resp. } \\
\text { bleomycin }+ \\
\text { electroporation } \\
\text { or bleomycin } \\
\text { alone }(P=.002)\end{array}$ & 36.5 months & $\begin{array}{l}\text { s No systemic adverse } \\
\text { events. Local: } \\
\text { electric shock } \\
\text { sensation, muscle } \\
\text { spasm, pain, } \\
\text { necrosis, and } \\
\text { eschar }\end{array}$ & $\begin{array}{l}\text { Funded in } \\
\text { part by } \\
\text { Genetronic } \\
\text { Inc, } \\
\text { San Diego, } \\
\text { CA, USA }\end{array}$ \\
\hline
\end{tabular}




\begin{tabular}{|c|c|c|c|c|c|c|c|c|c|c|c|c|c|}
\hline $\begin{array}{l}\text { First author, } \\
\text { year }\end{array}$ & $\begin{array}{c}\text { Type of } \\
\text { lesion }\end{array}$ & $\begin{array}{l}\text { Study } \\
\text { design }\end{array}$ & $\begin{array}{c}\begin{array}{c}\text { No. of } \\
\text { patients } \\
\text { (lesions) }\end{array} \\
\end{array}$ & Intervention & Anesthetics & Frequency & $\begin{array}{c}\text { Concentration } \\
\text { \& dosage }\end{array}$ & $\begin{array}{l}\text { Comparative } \\
\text { intervention }\end{array}$ & $\begin{array}{c}\text { Result per } \\
\text { patient }\end{array}$ & $\begin{array}{c}\text { Result per } \\
\text { lesion }\end{array}$ & $\begin{array}{l}\text { Follow- } \\
\text { up time }\end{array}$ & Adverse events & $\begin{array}{l}\text { Conflicts of } \\
\text { interest/ } \\
\text { funding }\end{array}$ \\
\hline $\begin{array}{r}\text { Marty, }{ }_{1}^{39} \\
2006\end{array}$ & $\begin{array}{l}\text { Cutaneous } \\
\text { metastases of } \\
\text { melanoma and } \\
\text { nonmelanoma } \\
\text { cancer }\end{array}$ & CCT & $62(290)$ & $\begin{array}{l}\text { Intralesional } \\
\text { bleomycin } \\
\text { injection, } \\
\text { followed by } \\
\text { electroporation }\end{array}$ & $\begin{array}{l}\text { Local anesthetic } \\
\quad \text { injection } \\
\text { (lidocaine 2\% + } \\
\text { epinephrine) } \\
\text { or general } \\
\text { anesthesia } \\
\text { (propofol + } \\
\text { remifentanil) }\end{array}$ & 1 treatment & $\begin{array}{l}1 \mathrm{U} / \mathrm{mL}, \\
0.25-1 \mathrm{U} / \mathrm{cm}^{3} \\
\text { depending on } \\
\text { tumor size. }\end{array}$ & $\begin{array}{l}\text { Intralesional } \\
\text { cisplatin } \\
(2 \mathrm{mg} / \mathrm{mL}, \\
\left.0.5-2 \mathrm{mg} / \mathrm{cm}^{3}\right) \\
\text { or intravenous } \\
\text { bleomycin } \\
\left(15 \mathrm{U} / \mathrm{m}^{2}\right), \\
\text { all followed by } \\
\text { electroporation }\end{array}$ & $\begin{array}{l}\text { OR for all nodules } \\
\text { per patient } 63.4 \% \\
\text { (intralesional and } \\
\text { intravenous } \\
\text { bleomycin and } \\
\text { intralesional } \\
\text { cisplatin) }\end{array}$ & $\begin{array}{l}\text { CR per nodule } \\
88.2 \%, 73.1 \% \text {, } \\
\text { and } 75.4 \% \\
\text { resp. bleomycin } \\
\text { intravenous, } \\
\text { bleomycin } \\
\text { intralesional } \\
\text { or cisplatin } \\
\text { intralesional, } \\
\text { all followed by } \\
\text { electroporation } \\
(P=.09)\end{array}$ & \multirow{2}{*}{\multicolumn{2}{|c|}{$\begin{array}{c}\text { 60-380 days No systemic adverse } \\
\text { events. Local: } \\
\text { pain and muscle } \\
\text { contraction }\end{array}$}} & $\begin{array}{l}\text { Not } \\
\quad \text { reported }\end{array}$ \\
\hline \multicolumn{12}{|l|}{$\begin{array}{l}\text { Keloids and } \\
\text { hypertrophic } \\
\text { scars }\end{array}$} & & \\
\hline $\begin{array}{l}\text { Kabel, } \\
2016^{14}\end{array}$ & $\begin{array}{l}\text { Keloids and } \\
\text { hypertrophic } \\
\text { scars }\end{array}$ & CCT & $120(120)$ & $\begin{array}{l}\text { ) Intralesional } \\
\text { bleomycin } \\
\text { injection }\end{array}$ & $\begin{array}{l}\text { Local } \\
\text { anesthetic } \\
\text { injection } \\
\text { (mepivacaine } \\
\text { HCl 3\%) }\end{array}$ & $\begin{array}{r}2-6 \times \text {, interval } \\
\text { of } 2 \text { weeks }\end{array}$ & $\begin{array}{l}1.5 \mathrm{U} / \mathrm{mL} \\
0.5-1 \mathrm{~mL} / \mathrm{cm}^{2} \\
\leq 4 \mathrm{~mL} \text { per } \\
\text { treatment }\end{array}$ & $\begin{array}{l}\text { II: Intralesional } \\
\text { 5-FU } 50 \mathrm{mg} / \mathrm{mL} \text {. } \\
\text { III: intralesional } \\
\text { 5-FU } 0.9 \mathrm{~mL} \\
\text { of } 50 \mathrm{mg} / \mathrm{mL}+ \\
\text { TCA } 0.1 \mathrm{~mL} \\
\text { of } 40 \mathrm{mg} / \mathrm{mL}\end{array}$ & $\begin{array}{l}\text { VSS mean } \\
\text { improvement } \\
\text { of } 73 \% \text { vs 54\% } \\
\text { vs 55\%, resp. } \\
\text { bleomycin or } \\
5 \text {-FU or } 5-\mathrm{FU}+ \\
\text { TCA. }(P<.05) \\
\text { No. of } \\
\text { treatments } \\
\text { required } 2-6 \text { vs } \\
4-6 \text { vs } 5-6 \text { resp. } \\
\text { bleomycin vs } \\
5-\mathrm{FU} \text { or } 5-\mathrm{FU}+ \\
\text { TCA }(P<.05) . \\
\text { Recurrence rate } \\
0 \% \text { vs } 40 \% \\
\text { vs } 46.67 \%\end{array}$ & Not reported & 12 months & $\begin{array}{l}\text { No systemic adverse } \\
\text { events. Local: } \\
\text { hyperpigmentation, } \\
\text { pain, and } \\
\text { ulceration }\end{array}$ & $\begin{array}{l}\text { None } \\
\text { declared }\end{array}$ \\
\hline $\begin{array}{l}\text { Payapvipapong, }{ }^{2} \\
2015\end{array}$ & $\begin{array}{l}{ }^{0} \text { Keloids and } \\
\text { hypertrophic } \\
\text { scars } \\
\text { (Fitzpatrick } \\
\text { skin types III } \\
\text { to V) }\end{array}$ & RCT & $26(26)$ & $\begin{array}{l}\text { Intralesional } \\
\text { bleomycin } \\
\text { injection }\end{array}$ & None & $\begin{array}{l}3 \times \text {, interval } \\
\text { of } 4 \text { weeks }\end{array}$ & $\begin{array}{l}1 \mathrm{U} / \mathrm{mL} \\
\quad \leq 6 \mathrm{~mL} \text { per } \\
\text { treatment }\end{array}$ & $\begin{array}{l}\text { Intralesional } \\
\text { TCA injection, } \\
10 \mathrm{mg} / \mathrm{mL}\end{array}$ & $\begin{array}{l}\text { (1) POSAS } \\
\text { improvement } \\
38.1 \% \text { vs } 27.5 \% \text {, } \\
\text { and reported } \\
\text { very good } \\
\text { improvement } \\
50 \% \text { vs } 50 \% \\
\text { resp. bleomycin } \\
\text { or TCA. (No } \\
\text { statistical } \\
\text { difference.) } \\
\text { (2) Improvement } \\
\text { evaluated by } \\
\text { ultrasonography } \\
30 \% \text { vs } 46 \% \text { resp. } \\
\text { bleomycin or }\end{array}$ & Not reported & $\begin{array}{l}\text { Not } \\
\quad \text { reported }\end{array}$ & $\begin{array}{l}\text { No systemic adverse } \\
\text { events. Local for } \\
\text { bleomycin } \\
\text { injection: } \\
\text { Hyperpigmentation, } \\
\text { pruritus, pain, } \\
\text { burning sensation } \\
\text { and vesicle-bullae } \\
\text { formation. Local } \\
\text { for TAC injection: } \\
\text { pruritus, pain, } \\
\text { and skin atrophy }\end{array}$ & $\begin{array}{l}\text { None } \\
\text { declared }\end{array}$ \\
\hline
\end{tabular}




\begin{tabular}{|c|c|c|c|c|c|c|c|c|c|c|c|c|}
\hline & & & & & & & & $\begin{array}{l}\text { TCA. (No } \\
\text { statistical } \\
\text { difference.). } \\
\text { (3) Photograph } \\
\text { evaluation by } 3 \\
\text { dermatologists: } \\
\text { no difference }\end{array}$ & & & & \\
\hline $\begin{array}{c}\text { Naeini, }{ }^{40} \\
2006\end{array}$ & $\begin{array}{l}\text { Keloids and } \\
\text { hypertrophic } \\
\text { scars }\end{array}$ & CCT 45 (45) & $\begin{array}{l}\text { Intralesional } \\
\text { bleomycin } \\
\text { injection } \\
\text { administered } \\
\text { with } \\
\text { multipuncture } \\
\text { technique }\end{array}$ & $\begin{array}{l}\text { Local anesthetic } \\
\text { injection (2\% } \\
\text { lidocaine) }\end{array}$ & $\begin{array}{l}4 \times \text {, interval } \\
\text { of } 1 \text { month }\end{array}$ & $\begin{array}{l}1.5 \mathrm{U} / \mathrm{mL}, \\
\leq 2 \mathrm{~m} / \mathrm{cm}^{2} \\
\text { and } \leq 10 \mathrm{U} \\
\text { of bleomycin } \\
\text { per } \\
\text { treatment } \\
\text { session }\end{array}$ & $\begin{array}{l}\text { Cryotherapy } \\
\text { (1 freeze-thaw } \\
\text { cycle) } \\
\text { intralesional } \\
\text { TCA injections } \\
40 \mathrm{mg} / \mathrm{mL} \text {, } \\
0.1-1 \mathrm{~mL} \text {. }\end{array}$ & $\begin{array}{l}\text { Mean resolution } \\
\text { score 88.3\% } \\
\text { vs } 87.3 \% \text { resp. } \\
\text { bleomycin or } \\
\text { cryotherapy }+ \\
\text { TCA } P=.001) . \\
>100 \mathrm{~mm}^{2} \\
\text { lesions had } \\
\text { significant } \\
\text { better therapeutic } \\
\text { response } \\
\text { with bleomycin } \\
(P=.031 . \\
\text { Disappearance } \\
\text { of pain, } \\
\text { tenderness, or } \\
\text { pruritus } 69 \% \text { vs } \\
\text { 49\% resp. } \\
\text { bleomycin } \\
\text { or cryotherapy }+ \\
\text { TCA. Recurrence } \\
\text { rate e } \% \text { for both } \\
\text { groups }\end{array}$ & Not reported & 3 months & $\begin{array}{l}\text { No systemic adverse } \\
\text { events. Local } \\
\text { reaction after } \\
\text { bleomycin: } \\
\text { hyperpigmentation. } \\
\text { Local reaction after } \\
\text { cryotherapy + } \\
\text { TCA: } \\
\text { hypopigmentation } \\
\text { and telangiectasia. } \\
\text { Treatment related } \\
\text { pain not reported. }\end{array}$ & $\begin{array}{l}\text { None } \\
\text { declared } \\
\text { n. } \\
\text { r. }\end{array}$ \\
\hline $\begin{array}{c}\text { Hemangioma } \\
\text { Pandey, } \text {, }_{11} \\
2018\end{array}$ & $\begin{array}{l}\text { Infantile } \\
\text { hemangioma } \\
\text { (not } \\
\text { responding } \\
\text { to propranolol) }\end{array}$ & $\begin{array}{l}\text { RCT } \quad 67(67) \\
\text { ) }\end{array}$ & $\begin{array}{l}\text { Intralesional } \\
\text { bleomycin } \\
\text { injection }\end{array}$ & Not reported & $\begin{array}{r}4-6 \times \text {, interval } \\
\text { of } 4 \text { weeks }\end{array}$ & $\begin{array}{l}\text { Concentration } \\
\text { not reported, } \\
0.5 \text { U/kg per } \\
\text { treatment, } \\
\leq 15 \cup \text { per } \\
\text { single dose }\end{array}$ & $\begin{array}{l}\text { Intralesional } \\
\text { TCA } 2 \mathrm{mg} / \mathrm{kg}\end{array}$ & $\begin{array}{l}\text { Excellent response } \\
\text { rate per patient } \\
47.2 \% \text { v } 25.8 \% \text {, } \\
\text { PR } 44.4 \% \text { vs } \\
48.4 \%, \text { no } \\
\text { response } 8.3 \% \text { vs } \\
25.8 \% \text { ress. } \\
\text { bleomycin or TCA } \\
(P=.074) \text {. } \\
\text { Bleomycin has } \\
\text { better response } \\
\text { rates than TCA in } \\
\text { nonresponders } \\
\text { to propranolol } \\
\text { (50\% vs } 7.7 \% \text {, } \\
P=.037)\end{array}$ & Not reported & $\begin{array}{c}7.42-9.38 \\
\text { months }\end{array}$ & $\begin{array}{l}\text { No systemic } \\
\text { adverse events. } \\
\text { Local: superficial } \\
\text { ulcer. Pain not } \\
\text { reported }\end{array}$ & $\begin{array}{l}\text { None } \\
\text { declared }\end{array}$ \\
\hline
\end{tabular}


Table II. Cont'd

\begin{tabular}{|c|c|c|c|c|c|c|c|c|c|c|c|c|c|}
\hline $\begin{array}{l}\text { First author, } \\
\text { year }\end{array}$ & $\begin{array}{c}\text { Type of } \\
\text { lesion }\end{array}$ & $\begin{array}{l}\text { Study } \\
\text { design }\end{array}$ & $\begin{array}{c}\begin{array}{c}\text { No. of } \\
\text { patients } \\
\text { (lesions) }\end{array} \\
\end{array}$ & Intervention & Anesthetics & Frequency & $\begin{array}{c}\text { Concentration } \\
\& \text { dosage }\end{array}$ & $\begin{array}{l}\text { Comparative } \\
\text { intervention }\end{array}$ & $\begin{array}{c}\text { Result per } \\
\text { patient }\end{array}$ & $\begin{array}{c}\text { Result per } \\
\text { lesion }\end{array}$ & $\begin{array}{l}\text { Follow- } \\
\text { up time }\end{array}$ & Adverse events & $\begin{array}{c}\text { Conflicts of } \\
\text { interest/ } \\
\text { funding }\end{array}$ \\
\hline $\begin{array}{c}\text { Thayal, }{ }^{42} \\
2012\end{array}$ & $\begin{array}{l}\text { Infantile } \\
\text { hemangiomas }\end{array}$ & $\mathrm{CCT}$ & $20(20)$ & $\begin{array}{l}\text { Intralesional } \\
\text { bleomycin } \\
\text { injection }\end{array}$ & $\begin{array}{l}\text { General } \\
\quad \text { anesthesia }\end{array}$ & $\begin{array}{l}1-3 \times \text {, interval } \\
\text { of } 6 \text { weeks }\end{array}$ & $\begin{array}{l}\text { Concentration } \\
\text { not } \\
\text { reported, } \\
0.5 \mathrm{U} / \mathrm{kg} \\
\text { body } \\
\text { weight per } \\
\text { treatment, } \\
\leq 12 \mathrm{U} \text { per } \\
\text { treatment }\end{array}$ & $\begin{array}{l}\text { Propranolol (oral) } \\
0.16-2 \mathrm{mg} / \mathrm{kg} \\
\text { body weight } \\
\text { per day for } \\
6 \text { weeks }\end{array}$ & $\begin{array}{l}\text { Volume reduction of } \\
75 \%-90 \% \text { after } \\
5 \text { months: } \\
62.5 \% \text { vs } 60 \% \text {, } \\
\text { overall size } \\
\text { reduction } 80 \% \text { vs } \\
85 \% \text { resp. } \\
\text { bleomycin or oral } \\
\text { propranolol }\end{array}$ & Not reported & 5 months & $\begin{array}{l}\text { No systemic adverse } \\
\text { events. Local: } \\
\text { febrile episode, } \\
\text { superficial } \\
\text { ulceration and } \\
\text { raised alkaline } \\
\text { phosphatase. } \\
\text { Pain not reported }\end{array}$ & $\begin{array}{l}\text { Not } \\
\quad \text { reported }\end{array}$ \\
\hline \multicolumn{14}{|c|}{$\begin{array}{l}\text { Other } \\
\text { indications }\end{array}$} \\
\hline $\begin{array}{l}\text { Lee }^{43} \\
\quad 2014\end{array}$ & Corns & CCT & $50(64)$ & $\begin{array}{l}\text { Intralesional } \\
\text { bleomycin } \\
\text { injection }\end{array}$ & $\begin{array}{l}\text { Bleomycin } \\
\text { diluted } \\
\text { with } 2 \% \\
\text { lidocaine }\end{array}$ & $\begin{array}{l}\text { 3-10X, interval } \\
\text { of } 3 \text { weeks }\end{array}$ & $\begin{array}{c}1 \mathrm{U} / \mathrm{mL}_{,} \leq 2 \mathrm{U} \\
\text { per } \\
\text { treatment }\end{array}$ & $\begin{array}{c}\text { Only callus } \\
\text { paring }\end{array}$ & $\begin{array}{l}\text { CR per patient } 37 \% \\
\text { vs } 7 \% \text {, } \\
\text { excellent/good } \\
\text { response in pain } \\
\text { reduction } 86 \% \text { vs } \\
47 \% \text { resp. } \\
\text { bleomycin or } \\
\text { callus paring } \\
\text { (statistically } \\
\text { significant) }\end{array}$ & $\begin{array}{l}>50 \% \text { decrease } \\
\text { in size in } 80 \% \\
\text { vs } 38 \% \text { resp. } \\
\text { bleomycin or } \\
\text { callus paring. } \\
\text { Recurrence } \\
\text { rate per lesion } \\
38 \% \text { vs } 67 \% \\
\text { resp. bleomycin } \\
\text { or paring }\end{array}$ & 3 months & $\begin{array}{l}\text { No systemic adverse } \\
\text { events. Local: } \\
\text { blackening, } \\
\text { eschar formation } \\
\text { and pain }\end{array}$ & $\begin{array}{l}\text { None } \\
\quad \text { declared }\end{array}$ \\
\hline $\begin{array}{l}\text { Roberts, }{ }^{44} \\
1980\end{array}$ & VIN & CCT & $12(12)$ & $\begin{array}{l}\text { Intralesional } \\
\text { bleomycin } \\
\text { injection }\end{array}$ & None & $\begin{array}{l}\text { Once weekly } \\
\text { until pain } \\
\text { or ulceration } \\
\text { required } \\
\text { treatment } \\
\text { termination }\end{array}$ & $\begin{array}{l}1 \mathrm{U} / \mathrm{mL} \\
0.3-0.5 \mathrm{U} \\
\text { per treatment, } \\
\leq 19.9 \mathrm{U} \\
\text { total dose }\end{array}$ & $\begin{array}{l}\text { Topical bleomycin } \\
5 \% \text { solution } \\
\text { applied twice } \\
\text { daily for max. } \\
21 \text { days }\end{array}$ & $\begin{array}{l}\text { Remission rate } 20 \% \\
\text { vs } 0 \% \text {, disease } \\
\text { progression } \\
50 \% \text { vs } 57 \% \\
\text { resp. bleomycin } \\
\text { injection or topical } \\
\text { bleomycin }\end{array}$ & Not reported & $\begin{array}{l}\text { Not } \\
\quad \text { reported }\end{array}$ & $\begin{array}{l}\text { No systemic adverse } \\
\text { events. Local: } \\
\text { vulvar } \\
\text { erythema, vulvitis, } \\
\text { pain, ulceration, } \\
\text { dark discoloration }\end{array}$ & $\begin{array}{l}\text { Not } \\
\quad \text { reported }\end{array}$ \\
\hline
\end{tabular}

5-FU, 5-fluorouracil; $B C C$, basal cell carcinoma; $C C T$, clinical controlled trial; $C R$, complete response; $E C T$, electrochemotherapy; OR, overall response (CR + PR); $P O S A S$, Patient and Observer Scar Assessment Scale; $P R$, partial response; $R C T$, randomized controlled trial; $R R$, relative risk; $S C C$, squamous cell carcinoma; TCA, Triamcinolone acetonide; $U$, United States Pharmacopeia unit; VAS, visual analog scale; VIN, vulvar intraepithelial neoplasia; VSS, Vancouver Scar Scale.

* 16 biphasic pulses of $25-+25-\mu$ s duration, spaced at $0.6 \mathrm{~cm}$ with a duration-number product of 0.8 milliseconds and a total sequence duration of 9.6 milliseconds.

${ }^{\dagger} 16$ biphasic pulses of $50-+50-\mu$ s duration, spaced at 1.0 milliseconds with a duration-number product of 0.8 milliseconds and a total sequence duration of 7.1 milliseconds. 


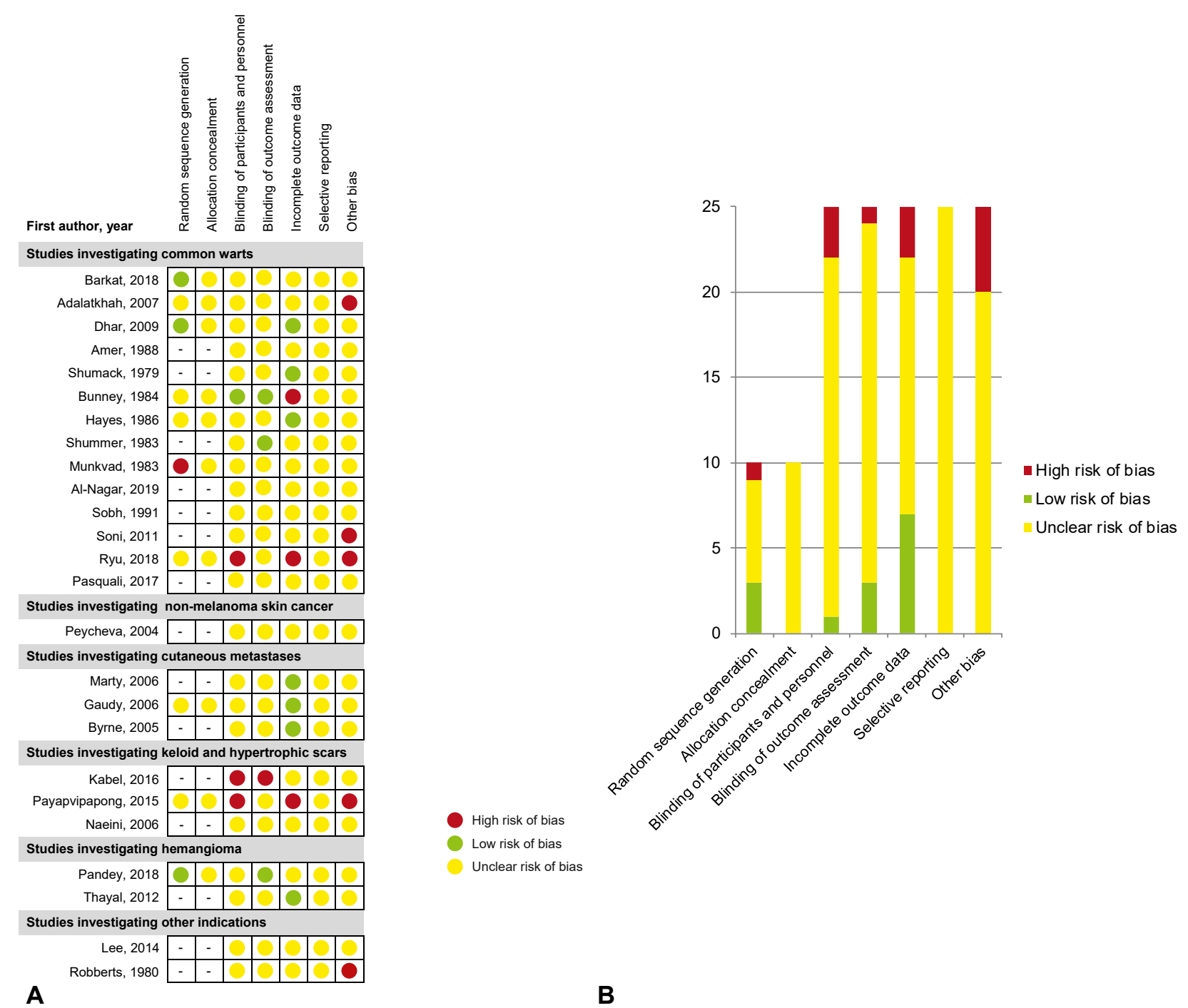

Fig 2. Methodologic quality of the included studies according to the Cochrane risk-of-bias assessment tool. (A) Methodologic quality of the included studies was categorized as high, low, or unclear risk of bias according to the Cochrane risk-of-bias assessment tool. Overall, methodologic quality was poor because of an unclear risk of bias for most of the assessed criteria. (B) Graph summarizing risk of bias of all 25 included studies.

indications. All included studies had a controlled study design, of which 10 were RCTs. Favorable efficacy outcomes were reported in 14 of 18 studies of intralesional bleomycin compared with placebo or other interventions. Methodologic quality was generally poor, with only 3 of 25 studies using appropriate randomization sequences and all studies lacking concealment of allocation. Furthermore, most studies had an unclear risk of bias for blinding and incomplete outcome data.

For all included studies, heterogeneity was found in treatment indication, use of anesthetics, treatment frequency and dosage, comparative intervention, outcome measures, and follow-up time. This heterogeneity precluded performing a meta-analysis.
Intralesional bleomycin treatment generally showed good results for common warts, keloids, hypertrophic scars, propranolol-resistant hemangioma, and corns. However, most studies focused on bleomycin treatment of common warts.

Recommendations for daily clinical practice are preferably based on adequately powered head-tohead clinical trials in which the new intervention is compared against another (gold standard) intervention. In common warts, intralesional bleomycin showed higher cure rates than cryotherapy. ${ }^{29,30,32}$ Plantar warts were more recalcitrant, possibly because of the endophytic character of the lesions leading to suboptimal drug delivery. ${ }^{19}$ In keloid and hypertrophic scars, intralesional 
bleomycin treatment resulted in better improvements than cryotherapy, followed by intralesional TCA, intralesional 5-FU/TCA injection, or intralesional 5-FU alone. ${ }^{14,40}$ In hemangioma, intralesional bleomycin was comparable to oral propranolol treatment but favorable to TCA injections in propranolol nonresponders. ${ }^{41,42}$ No head-to-head clinical trials are available for nonmelanoma skin cancer, cutaneous metastases, and vulvar intraepithelial neoplasia.

Topical treatment is a key element in dermatologic practice. Topical bleomycin administration showed poor results, however, because of the limited penetration of the lipophilic stratum corneum by this hydrophilic drug. ${ }^{44}$ Intralesional administration methods provided greater bleomycin bioavailability in the skin. Needle injections were most often used, but the microneedle patch and microneedling pen device may be the least painful techniques. ${ }^{32}$ One study using a mechanical jet injector reported poor response rates in common warts. This could be explained by spill of bleomycin solution due to suboptimal positioning of the device leading to insufficient dermal drug delivery. ${ }^{34}$

Five included studies reported outcomes of intralesional bleomycin, followed by electroporation (electrochemotherapy). ${ }^{33,36-39}$ Electroporation provides permeabilization of the lipophilic cell membrane, which enhances penetration of bleomycin into the cytosol and leads to increased cytotoxicity. ${ }^{45}$ Electrochemotherapy was primarily investigated in uncontrolled trials focusing on cutaneous malignancies. ${ }^{46}$ In all included studies, electrochemotherapy showed significant higher success rates than intralesional bleomycin alone for metastases of melanoma and common warts. ${ }^{33,37,38}$

Besides electroporation, local anesthetics have shown to change the cell permeabilization and increase cellular uptake of bleomycin in vitro. ${ }^{47}$ In clinical studies, adding anesthetics to the bleomycin solution led to higher complete cure rates in common warts than with bleomycin alone. ${ }^{23,28-31}$

\section{Strengths and limitations}

Strengths of this systematic review include the use of a comprehensive data search, inclusion of only controlled studies with appropriate sample sizes, reporting of outcome measures as effect size and adverse events, assessment of methodologic quality, and inclusion of off-label dermatologic indications.

Limitations of this review are that one-third of the studies were published before 2000 (1979-1994) with a lack of statistical analyses, all studies had an unclear risk of bias for most of the methodologic criteria, and several studies did not report the number of patients or lesions and follow-up time.

\section{Intralesional bleomycin treatment in clinical practice}

Treatment characteristics, such as efficacy, safety, tolerability, usability, and also patient preference are important factors considered in making a treatment choice. No systemic adverse events were reported after bleomycin treatment for any of the dermatologic indications, making this a relatively well-tolerated treatment. Local pain was reported during and after the procedure but was evidently well-tolerated, and cotreatment with local anesthetics was usually sufficient. With regard to usability, bleomycin is relatively easy to administer, requires minimal resources, is inexpensive, and is widely available, making it a treatment that can be easily incorporated into daily clinical practice.

Recalcitrant common warts and keloids are daily treated in our clinic with intralesional bleomycin. A reproducible success is seen using a mixture of bleomycin $(1 \mathrm{U} / \mathrm{mL})$ with lidocaine hydrochloride $(5 \mathrm{mg} / \mathrm{mL})$ in saline (unpublished data). In most cases, a conventional 30- or 33-gauge needle injection is used, with a maximum of $2 \mathrm{~mL}$ per treatment. The bleomycin solution should be deposited in the mid-dermal part of keloids to prevent necrosis or ulcerations. In warts, however, bleomycin should be injected in the superficial dermis to induce necrosis. Intralesional bleomycin treatment is well tolerated, but patients experience pain for an average of 2.5 days after the injection. A mechanical jet injector (eg, DermoJet; Akra, Pau, France) is also practical but has several disadvantages such as risk of aerosol formation and drug spill. To reduce the risk of bleomycin inhalation, a pair of wet gauzes is wrapped around the tip of the device, the operator and patient wear surgical respirator (3M, St Paul, MN) and safety glasses, and a laser smoke evacuator is used to capture any aerosols.

\section{CONCLUSIONS}

This review provides a systematically conducted overview and methodologic quality assessment of the efficacy and safety of intralesional bleomycin treatment for dermatologic indications. For all reviewed indications, there was insufficient evidence to provide firm recommendations for intralesional bleomycin treatment in clinical practice. However, this review suggests that intralesional bleomycin is a successful and well-tolerated treatment option for recalcitrant warts. 
Clearly, more head-to-head trials with high methodologic quality are needed to provide future evidence-based recommendations for daily clinical practice. New and less painful administration methods, such as laser-assisted drug delivery and needle-free pneumatic injection, should be investigated besides conventional injections for common warts, keloid, hypertrophic scars, and nonmelanoma skin cancer.

We thank the biomedical information specialists of Erasmus MC Rotterdam for their assistance in the electronic literature search.

\section{REFERENCES}

1. U.S. Food \& Drug Administration. Orphan-designated products with marketing approvals for both common and rare disease indications. Available at: https://www.fda.gov/ media/78808/download; 2019. Accessed March 11, 2019.

2. European Medicines Agency. Overall summary of the scientific evaluation of bleomycin pharmachemie and associated names. Available at: https://www.ema.europa.eu/en/documents/ referral/bleomycin-article-29-referral-annex-i-ii-iii_en.pdf; 2009. Accessed March 11, 2019.

3. Umezawa H, Maeda K, Takeuchi T, Okami Y. New antibiotics, bleomycin A and B. J Antibiot (Tokyo). 1966;19(5):200-209.

4. Onuma T, Holland JF, Sako K, Shedd DP. Effects of combination therapy with bleomycin (NSC-125066) and dibromodulcitol (NSC-104800) on squamous cell carcinoma in man. Cancer Chemother Rep. 1972;56(5):625-633.

5. Yamamoto T. Bleomycin and the skin. Br J Dermatol. 2006; 155(5):869-875.

6. Burger RM, Peisach J, Horwitz SB. Activated bleomycin. A transient complex of drug, iron, and oxygen that degrades DNA. J Biol Chem. 1981;256(22):11636-11644.

7. Tounekti O, Pron G, Belehradek J Jr, Mir LM. Bleomycin, an apoptosis-mimetic drug that induces two types of cell death depending on the number of molecules internalized. Cancer Res. 1993;53(22):5462-5469.

8. Hendricks T, Martens MF, Huyben CM, Wobbes T. Inhibition of basal and TGF beta-induced fibroblast collagen synthesis by antineoplastic agents. Implications for wound healing. $\mathrm{Br} J$ Cancer. 1993;67(3):545-550.

9. Samuels ML, Johnson DE, Holoye PY, Lanzotti VJ. Large-dose bleomycin therapy and pulmonary toxicity. A possible role of prior radiotherapy. JAMA. 1976;235(11):1117-1120.

10. D'Cruz D. Autoimmune diseases associated with drugs, chemicals and environmental factors. Toxicol Lett. 2000;112-113: 421-432.

11. Kerr LD, Spiera H. Scleroderma in association with the use of bleomycin: a report of 3 cases. J Rheumatol. 1992; 19(2):294-296.

12. Scallan PJ, Kettler AH, Levy ML, Tschen JA. Neutrophilic eccrine hidradenitis. Evidence implicating bleomycin as a causative agent. Cancer. 1988;62(12):2532-2536.

13. Altaykan A, Boztepe G, Erkin G, Ozkaya O, Ozden E. Acute generalized exanthematous pustulosis induced by bleomycin and confirmed by patch testing. J Dermatolog Treat. 2004; 15(4):231-234.

14. Kabel AM, Sabry HH, Sorour NE, Moharm FM. Comparative study between intralesional injection of bleomycin and 5 -fluorouracil in the treatment of keloids and hypertrophic scars. J Dermatol Dermatol Surg. 2016;20(1):32-38.
15. Abess A, Keel DM, Graham BS. Flagellate hyperpigmentation following intralesional bleomycin treatment of verruca plantaris. Arch Dermatol. 2003;139(3):337-339.

16. Epstein E. Intralesional bleomycin and Raynaud's phenomenon. J Am Acad Dermatol. 1991;24(5 Pt 1):785-786.

17. Gregg LJ. Intralesional bleomycin and Raynaud's phenomenon. J Am Acad Dermatol. 1992;26(2 Pt 1):279-280.

18. Miller RA. Nail dystrophy following intralesional injections of bleomycin for a periungual wart. Arch Dermatol. 1984;120(7): 963-964.

19. Amer M, Diab N, Ramadan A, Galal A, Salem A. Therapeutic evaluation for intralesional injection of bleomycin sulfate in 143 resistant warts. J Am Acad Dermatol. 1988;18(6):1313-1316.

20. Payapvipapong K, Niumpradit N, Piriyanand C, Buranaphalin S, Nakakes A. The treatment of keloids and hypertrophic scars with intralesional bleomycin in skin of color. J Cosmet Dermatol. 2015;14(1):83-90.

21. Sarihan H, Mocan H, Yildiz K, Abes M, Akyazici R. A new treatment with bleomycin for complicated cutaneous hemangioma in children. Eur J Pediatr Surg. 1997;7(3):158-162.

22. Stefanou A, Siderov J, Society of Hospital Pharmacists of Australia Committee of Specialty Practice in Oncology. Medical errors. Dosage nomenclature of bleomycin needs to be standardised to avoid errors. BMJ. 2001;322(7299):1423-1424.

23. Barkat MT, Abdel-Aziz RTA, Mohamed MS. Evaluation of intralesional injection of bleomycin in the treatment of plantar warts: clinical and dermoscopic evaluation. Int J Dermatol. 2018;57(12):1533-1537.

24. Shumack PH, Haddock MJ. Bleomycin: an effective treatment for warts. Australas J Dermatol. 1979;20(1):41-42.

25. Bunney MH, Nolan MW, Buxton PK. The treatment of resistant warts with intralesional bleomycin: a controlled clinical trial. $\mathrm{Br}$ J Dermatol. 1984;111(2):197-207.

26. Shumer SM, O'Keefe EJ. Bleomycin in the treatment of recalcitrant warts. J Am Acad Dermatol. 1983;9(1):91-96.

27. Sobh MA, Abd El-Razic MM, Rizc RA, Eid MM, Abd el-Hamid IA, Ghoneim MA. Intralesional injection of bleomycin sulphate into resistant warts in renal transplant recipients versus nontransplant warty patients. Acta Derm Venereol. 1991;71(1):6366.

28. Soni P, Khandelwal K, Aara N, Ghiya BC, Mehta RD, Bumb RA. Efficacy of intralesional bleomycin in palmo-plantar and periungual warts. J Cutan Aesthet Surg. 2011;4(3):188-191.

29. Adalatkhah $H$, Khalilollahi $H$, Amini N, Sadeghi-Bazargani $H$. Compared therapeutic efficacy between intralesional bleomycin and cryotherapy for common warts: a randomized clinical trial. Dermatol Online J. 2007;13(3):4.

30. Dhar SB, Rashid MM, Islam AZMM, Bhuiyan MSI. Intralesional bleomycin in the treatment of cutaneous warts: a randomized clinical trial comparing it with cryotherapy. Indian J Dermatol Venereol Leprol. 2009;75(3):262-267.

31. Al-Naggar MR, Al-Adl AS, Rabie AR, Abdelkhalk MR, Elsaie ML. Intralesional bleomycin injection vs microneedling-assisted topical bleomycin spraying in treatment of plantar warts. J Cosmet Dermatol. 2019;18(1):124-128.

32. Ryu HR, Jeong HR, Seon-Woo HS, et al. Efficacy of a bleomycin microneedle patch for the treatment of warts. Drug Deliv Transl Res. 2018;8(1):273-280.

33. Pasquali $P$, Freites-Martinez A, Gonzalez S, Spugnini EP, Baldi A. Successful treatment of plantar warts with intralesional bleomycin and electroporation: pilot prospective study. Dermatol Pract Concept. 2017;7(3):21-26.

34. Munkvad M, Genner J, Staberg B, Kongsholm H. Locally injected bleomycin in the treatment of warts. Dermatologica. 1983;167(2):86-89. 
35. Hayes ME, O'Keefe EJ. Reduced dose of bleomycin in the treatment of recalcitrant warts. J Am Acad Dermatol. 1986;15(5 Pt 1):1002-1006.

36. Peycheva E, Daskalov I. Electrochemotherapy of skin tumours: comparison of two electroporation protocols. J BUON. 2004; 9(1):47-50.

37. Gaudy C, Richard MA, Folchetti G, Bonerandi JJ, Grob JJ. Randomized controlled study of electrochemotherapy in the local treatment of skin metastases of melanoma. J Cutan Med Surg. 2006;10(3):115-121.

38. Byrne CM, Thompson JF, Johnston $\mathrm{H}$, et al. Treatment of metastatic melanoma using electroporation therapy with bleomycin (electrochemotherapy). Melanoma Res. 2005;15(1):45-51.

39. Marty M, Sersa G, Garbay JR, et al. Electrochemotherapy-an easy, highly effective and safe treatment of cutaneous and subcutaneous metastases: results of ESOPE (European Standard Operating Procedures of Electrochemotherapy) study. Eur J Cancer Suppl. 2006;4(11):3-13.

40. Naeini FF, Najafian J, Ahmadpour K. Bleomycin tattooing as a promising therapeutic modality in large keloids and hypertrophic scars. Dermatol Surg. 2006;32(8):1023-1029.

41. Pandey V, Tiwari P, Sharma SP, Kumar R, Singh OP. Role of intralesional bleomycin and intralesional triamcinolone therapy in residual haemangioma following propranolol. Int J Oral Maxillofac Surg. 2018;47(7):908-912.

42. Thayal PK, Bhandari PS, Sarin YK. Comparison of efficacy of intralesional bleomycin and oral propanolol in management of hemangiomas. Plast Reconstr Surg. 2012;129(4):733e-735e.

43. Lee WJ, Lee SM, Won $\mathrm{CH}$, et al. Efficacy of intralesional bleomycin for the treatment of plantar hard corns. Int J Dermatol. 2014;53(12):e572-e577.

44. Roberts JA, Watring WG, Lagasse LD. Treatment of vulvar intraepithelial neoplasia (VIN) with local bleomycin. Cancer Clin Trials. 1980;3(4):351-354.

45. Heller R, Jaroszeski MJ, Reintgen DS, et al. Treatment of cutaneous and subcutaneous tumors with electrochemotherapy using intralesional bleomycin. Cancer. 1998; 83(1):148-157.

46. Seyed Jafari SM, Jabbary Lak F, Gazdhar A, Shafighi M, Borradori L, Hunger RE. Application of electrochemotherapy in the management of primary and metastatic cutaneous malignant tumours: a systematic review and meta-analysis. Eur J Dermatol. 2018;28(3):287-313.

47. Mizuno S, Ishida A. Selective enhancement of bleomycin cytotoxicity by local anesthetics. Biochem Biophys Res Commun. 1982;105(2):425-431. 